\title{
Comparative Performance Analysis of Contourlet Transform using Non Subsampled Algorithm in Digital Image Processing
}

\author{
Deepak Kourav $^{1}$, Dr. UBS Chandrawat ${ }^{2}$ \\ Research scholar Dr. K.N. Modi University, Newai (Rajasthan) ${ }^{1}$ \\ Acropolis Institute of Technology \& Research, Indore (M.P.) ${ }^{2}$
}

\begin{abstract}
In this paper we have compared Nun Subsampled Contourlet Transform and Wavelet Transform in image denoising on the basis of PSNR, RMSE and SSIM. In order to remove the image noise more effectively an image denoising algorithm based on Non Subsampled Contourlet Transform (NSCT) is proposed. The noisy image is first decomposed into multi-scale and multi-directional subbands by NSCT, and direction subbands of each high-pass component is processed by the new threshold function which is obtained by the Bayes threshold that based on stratified noise estimation. During the reconstruction, the low-pass subband constructed image is further denoisied by the bilateral filtering in the spatial domain. Experimental results compare with the result of image denoising using wavelet transform. It demonstrates that the proposed method can improve de-noising performance.
\end{abstract}

Keywords: NSCT, PSNR, RMSE, SSIM.

\section{INTRODUCTION}

In the process of collection and transmission, the digital image is often affected by the noise, such as compression, denoising, feature extraction, and inverse problems. So it is necessary to reduce the image noise to improve the quality of the image. Many denoising methods have been developed over the year, there are two main classes of digital image denoising methods: one is processed in the transform domain, the other in a spatial domain. Spatial domain filtering method is to operate directly on the image pixels in the spatial domain. But operating on the image pixel directly will lead to the fuzzy Image edge and details. The bilateral filter [1] and the nonlocal means filter are examples of methods which define the filter kernel in the spatial domain. Tomasi proposed the bilateral filtering (BF) by introducing the weighted function. The bilateral filtering Retains more image details and continuity compared with the traditional spatial filtering method.

The transform domain method process the coefficients in the transform domain, there are many transform domain methods, such as Wavelet transform [2], Shearlet transform [3] and Contourlet transform[4]. Multiresolution Bilateral Filtering (MBF) for Image Denoising which is proposed In the literature [5] combine two popular filters for the two domains, An input image is decomposed into its detail and approximation subbands through wavelet decomposition, bilateral filtering is applied to the approximation subbands, at the same time wavelet Thresholding is applied to the detail subbands, But the two-dimensional wavelet transform can only extract information in two directions. Nonsubsampled Contourlet Transform (NSCT) [6] is a multi-directional and multi-scale decomposition. We propose a method based on Non-subsampled Contourlet Transform and Bilateral Filtering. The new Threshold function is used in the detail subbands.

\section{ALGORITHM}

\section{A. Nonsubsampled Contourlet Transform}

Your The nonsubsampled CT (Contour-let Transform) is a multi-scale and multi-directional change that is as of late created on the base of CT (Contour-let Transform). It is totally move invariance makes up the deficiency of the subsampled CT (Contour-let Transform) proposed by Do and Vetterli. NSCT should be acknowledged by the Pyramid scale decay and directional disintegration in view of nonsubsampled directional channel banks.

In this denoising algorithm we find high and low frequency decomposition coefficients from the input picture using NSCT. The estimated high frequency coefficients are denoise by adaptiven Bayesian filter in NSCT domain, the remaining low frequency coefficient will reconstruct and pass by bilateral filter.

1. Give Noisy Picture as Input.

2. Apply NSCT to the noisy picture. 
3. Now through the decomposition process extract the NSCT coefficients in to high frequency coefficient and low frequency coefficient.

4. Now high frequency coefficients are denoise by adaptive Bayesian filter in NSCT domain and we get high frequency subband picture.

5. Now low frequency coefficient will reconstruct and pass by bilateral filter and we get low frequency subband picture.

6. We can get denoise picture by adding high frequency subband picture and frequency subband picture.

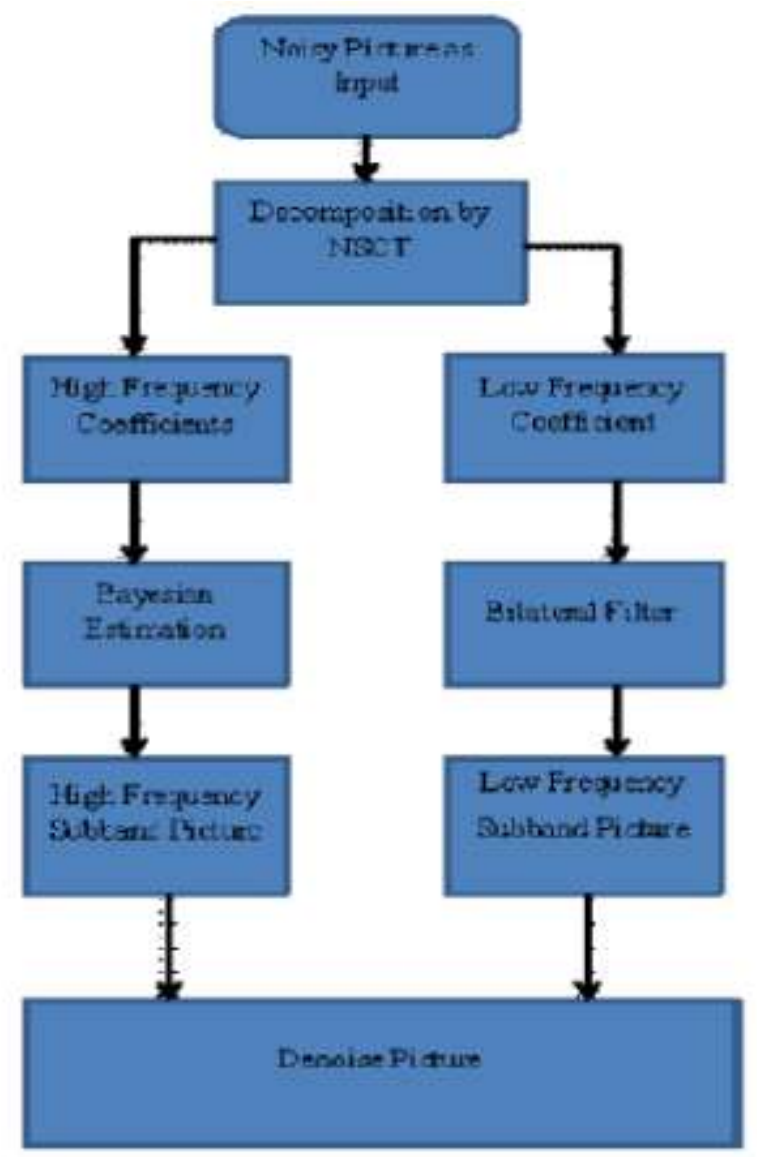

Fig.1 Flowchart of Proposed Algorithm

\section{B. Discrete Wavelet transform}

Wavelets are the scientific capacities which investigate information as indicated by the scale or determination. They help in concentrate a flag in various windows or in various resolutions. For instance, if the flag is seen in the substantial window, net element can be seen, and if seen in a little window, just the little components can be taken note. The wavelets give a few focal points more than Fourier changes. For example, they make an incredible showing with regards to in approximating signals with sharp spikes and flags having discontinuities. Wavelets can likewise display music, discourse, video and non-stationary stochastic signs. The wavelets can be utilized as a part of uses, for example, turbulence, picture pressure, human vision, seismic tremor forecast.

Noise in a Picture is either an additive or a multiplicative noise. Gaussian noise is generally called as additive white Gaussian noise it is equally distributed over the signal. Every pixel present in the noisy picture is the sum of the real pixel value and random Gaussian distributed noise value.

Addative noise can be represented as in eq

$$
w(x, y)=s(x, y)+n(x, y)
$$

Multiplicative noise can be represented as in eq

$$
\mathrm{w}(\mathrm{x}, \mathrm{y})=\mathrm{s}(\mathrm{x}, \mathrm{y}) \times \mathrm{n}(\mathrm{x}, \mathrm{y})
$$

In the above equation $\mathrm{w}(\mathrm{x}, \mathrm{y})$ represent the noisy picture, $\mathrm{s}(\mathrm{x}, \mathrm{y})$ represent the original picture and $\mathrm{n}(\mathrm{x}, \mathrm{y})$ represent the noise in the picture. The picture becomes noisy when noise is added in it. 
The denoising algorithm has the following steps:

1. First give the noisy picture as input.

2. Decomposition of picture using discrete wavelet transforms.

3. Calculate the threshold value of given picture.

4. Applying soft Thresholding on noisy pixels.

5. Now invert the decomposition using IDWT and recover the Picture.

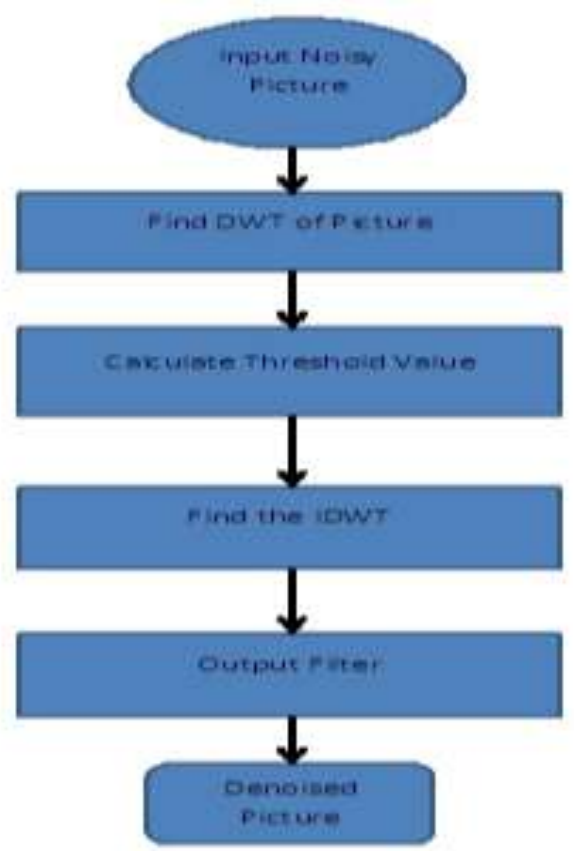

Fig. 2 Flowchart of denoising algorithm using DWT

\section{III.RESULT AND DISCUSSION}

This section represents the wavelet and contourlet result in the form of images and compare the PSNR, RMSE and SSIM in tabular form. From the result we can observe that the value of PSNR and SSIM is better for contourlet as compare to wavelet, where as the value of RMSE is greater for wavelet as compare to contourlet.

We can observe the quality of restored image by watching the snapshot carefully. In this section we have used images of Lena, Barbara, Pepper, and Car also find the values of PSNR, RMSE and SSIM in wavelet and contourlet domain and compare each result in the tabular form. We have also compare the snapshot of original noisy and denoise images in wavelet and contourlet domain

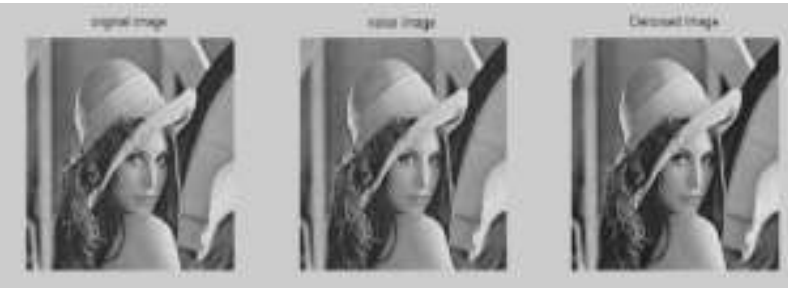

Fig 3 Barbara.jpg original, noisy and denoised image for $\sigma=0.1$ by wavelet transform

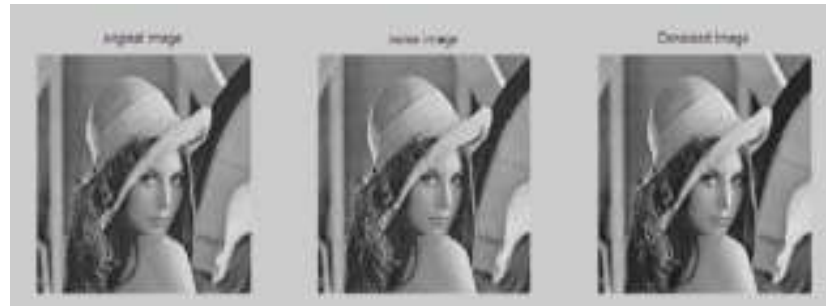

Fig 4 Barbara.jpg original, noisy and denoised image for $\sigma=0.1$ by contourlet transform 


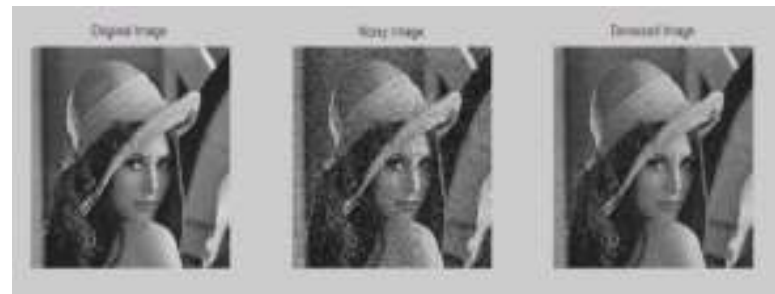

Fig 5 Barbara.jpg original, noisy and denoised image for $\sigma=0.25$ by wavelet transform

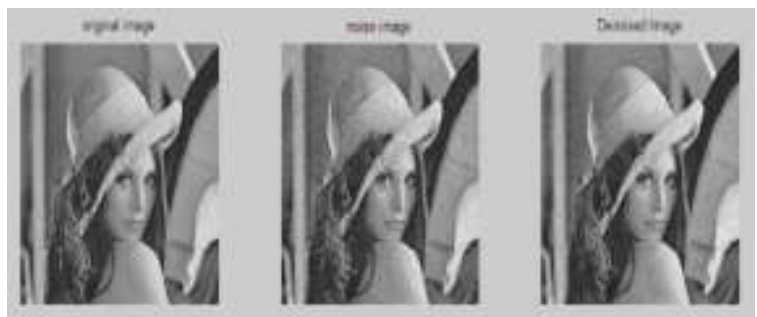

Fig 6 Barbara.jpg original, noisy and denoised image for $\sigma=0.25$ by contourlet transform

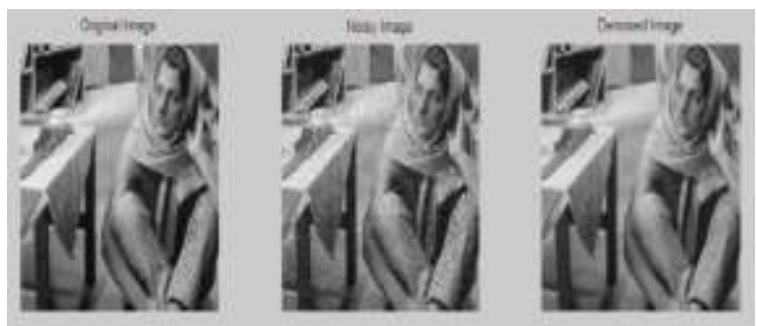

Fig 7 Barbara.jpg original, noisy and denoised image for $\sigma=0.1$ by wavelet transform

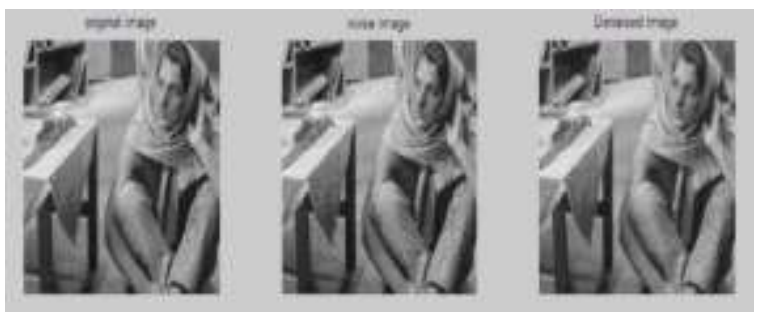

Fig 8 Barbara.jpg original, noisy and denoised image for $\sigma=0.1$ by contourlet transform

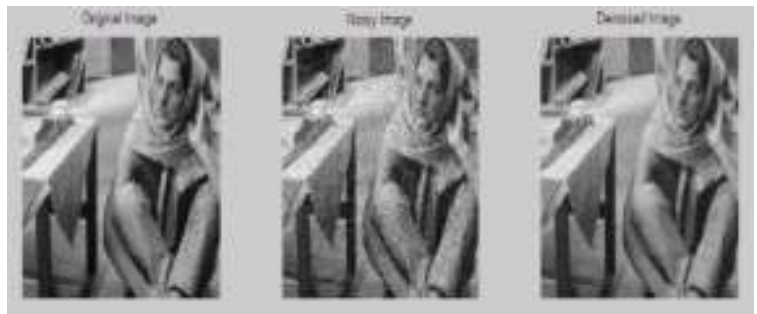

Fig 9 Barbara.jpg original, noisy and denoised image for $\sigma=0.25$ by wavelet transform

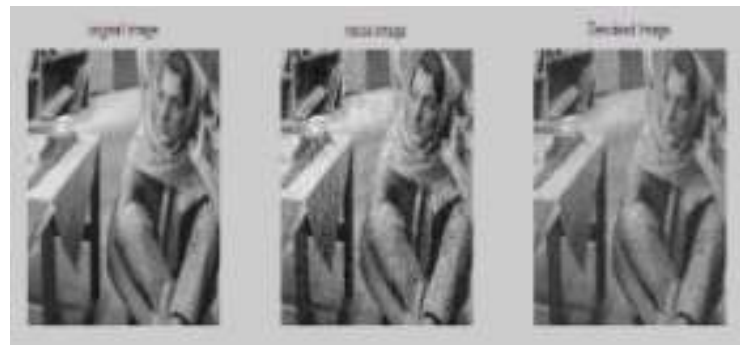

Fig 10 Barbara.jpg original, noisy and denoised image for $\sigma=0.25$ by contourlet transform 
Table.1 Comparitive analysis of Contourlet and DWT D for Lena and Barbara

\begin{tabular}{|l|l|l|l|l|l|l|}
\hline Transform & Input Image & Value of N & $\sigma$ & RMSE & PSNR & SSIM \\
\hline DWT & Lena.jpg & 512 & .1 & 8.00 & 30.10 & 0.9931 \\
\hline Contourlet & Lena.jpg & 512 & .1 & 6.3759 & 32.0400 & 0.9974 \\
\hline DWT & Lena.jpg & 512 & .25 & 11.2015 & 26.0010 & 0.9889 \\
\hline Contourlet & Lena.jpg & 512 & .25 & 9.9292 & 28.1925 & 0.9937 \\
\hline DWT & barbara.jpg & 512 & .1 & 15.7012 & 24.3025 & 0.9880 \\
\hline Contourlet & barbara.jpg & 512 & .1 & 14.2022 & 25.0837 & 0.9890 \\
\hline \multicolumn{1}{|c|}{ DWT } & barbara.jpg & 512 & .25 & 18.9265 & 21.6234 & 0.9848 \\
\hline Contourlet & barbara.jpg & 512 & .25 & 15.0389 & 24.5865 & 0.9865 \\
\hline
\end{tabular}

\section{IV.CONCLUSION}

In this paper we have proposed NSCT for image denoising and compare the result with DWT and we have observed that the performance of NSCT is better than the DWT on the basis of PSNR, RMSE and SSIM.

\section{ACKNOWLEDGMENT}

The heading of the Acknowledgment section and the References section must not be numbered. Causal Productions wishes to acknowledge Michael Shell and other contributors for developing and maintaining the IEEE LaTeX style files which have been used in the preparation of this template

\section{REFERENCES}

[1] Tomasi C., Manduchi R., Bilateral filtering for gray and color images. In Proceeding of the 1998 IEEE International Conference on Computer Vision, Bombay, India, pp. 839-846, 1998.

[2] Weyrich N., Warhola G. T., Wavelet shrinkage and generalized cross validation for image denoising. IEEE Transactions on Image Proceeding, 7 (1), pp. 82-90, 1998.

[3] Do M N, Vetterli M.,The Contourlet Transform: An Efficient Directional Multireslution Image Representation. IEEE Trans. on Image Processing, 14(12), pp. 2091-2106, 2005.

[4] Guo K., Labate D., Optimally Sparse Multidimensional Representation using Shearlets. SIAM J Math Anal, 39( 1), pp. 298-318, 2007.

[5] M. Zhang, Gunturk B. K., Multi-resolution bilateral filtering for image denoising. IEEE Trans on Image Processing, 17(12), pp. 2324-2333, 2008.

[6] Cunha A L, J.P. Zhou, Do M.N., The Non-subsampled Contourlet: Theory. Design and Applications. IEEE Trans. on Image Processing, 15(10), pp. 1779-1793, 2006

[7] L. Ding, L.J. Wang, Z.H. Jia, Adaptive Image Denoising Algorithm Integrating Bayes with Contourlet Transform. Laser Journal, 34(6), pp. 43$45,2013$.

[8] L. Li, H.X. Yang, Adaptive Threshold De-noising Algorithm Combined with Fourier Transform and Non-subsampled Contourlet Transform. Computer Engineering,38(17), pp. 238-241, 2012.

[9] Z.Q. Zhang, W.Y. Wang, A Modified Bilateral Filtering Algorithm. Journal of Image and Graphics, 14(3), pp. 443-447, 2009.

[10] N.N. Li, J.J. Cao, B. Li et al. Reweighted Global Bilateral Filtering Based on Normal Regularization. Journal of Computer-Aided Design \& Computer Graphics , 26(3), pp. 370-377, 2014.

[11] F. Tang, H.X. Yang, Y.W. Zeng, et al, Image denosing algorithm based on improved Contourlet transform. Computer Engineering and Applications, 50(5), pp. 132-136, 2014.

[12] Y. Yang, W.J. Zheng, S.Y. Huang, et al, Multi-focus image fusion based on human visual perception characteristic in Non-subsampled Contourlet transform domain. Journal of Image and graphics, 19(3), pp. 447-455, 2014. 